\title{
Apicomplexa primers amplify Proteromonas (Stramenopiles, Slopalinida, Proteromonadidae) in tissue and blood samples from lizards
}

\author{
João P.M.C. Maia ${ }^{1,2,3}{ }^{*}$, Elena Gómez-Díaz ${ }^{3}$ and D. James Harris ${ }^{1}$ \\ ${ }^{1}$ CIBIO, Centro de Investigação em Biodiversidade e Recursos Genéticos, Campus Agrário de Vairão, 4485-661 Vairão, Portugal; \\ ${ }^{2}$ Departamento de Biologia, Faculdade de Ciências, Universidade do Porto, Rua do Campo Alegre FC4, 4169-007 Porto, Portugal; \\ ${ }^{3}$ Institut de Biologia Evolutiva (CSIC-UPF), Passeig Marítim de la Barceloneta, 37-49. 08003 Barcelona, Spain
}

\begin{abstract}
Microscopy has traditionally been the most common method in parasitological studies, but in recent years molecular screening has become increasingly frequent to detect protozoan parasites in a wide range of vertebrate hosts and vectors. During routine molecular screening of apicomplexan parasites in reptiles using the 18S rRNA gene, we have amplified and sequenced Proteromonas parasites from three lizard hosts (less than $1 \%$ prevalence). We conducted phylogenetic analysis to confirm the taxonomic position and infer their relationships with other stramenopiles. Although our phylogeny is limited due to scarcity of molecular data on these protists, our results confirm they are closely related to Proteromonas lacertae. Our findings show that unexpected parasites can be amplified from host samples (blood and tissue) using general procedures to detect hemoparasites, and stress that positive PCR amplifications alone should not be considered as definitive proof of infection by particular parasites. Further validation by sequence confirmation and thorough phylogenetic assessment will not only avoid false positives and biased prevalence estimates but also provide valuable information on the biodiversity and phylogenetic relationships of other parasitic organisms. More generally, our results illustrate the perils of general diagnosis protocols in parasitological studies and the need of cross-validation procedures.
\end{abstract}

\section{Keywords}

Lizard, hemogregarine, 18S rRNA gene, molecular screening, microscopy, hemoparasites

\section{Introduction}

Traditionally, microscopy has been the gold-standard in parasitological studies and-there has been considerable effort to identify and classify the diversity of protist biota in reptiles, namely intestinal flagellates (e.g., Wood 1935; Wenrich 1947; Janakidevi 1961a, b; Krishnamurthy 1968; Telford 1970; Dollahon and Janovy 1971; Saratchandra and Narasimhamurti 1980a, b; Telford and Bursey 2003) and hemoparasites (e.g., Amo et al. 2005, Austin and Perkins 2006, Roca and Galdón 2010). Recently, molecular methods have been increasingly used for the detection of protists in both wild and domestic animals. Compared to more traditional approaches, such as microscopy, molecular methods benefit from good specificity and sensitivity in detecting parasitic infections (e.g., Rubini et al. 2005, Merino et al. 2009), especially at low parasitemia levels (Moody 2002), they are straightforward and are relatively low cost and not time consuming, thus allowing the screening of large numbers of samples in a reproducible manner. Moreover, molecular data provides additional and valuable information on the diversity of parasitic organisms and their genetic relationships. However, some studies estimate infection prevalence solely based on PCR screening without sequence confirmation (e.g., Ujvari et al. 2004, Vardo et al. 2005), which could lead to biased estimates since other related or unrelated organisms may be amplified. In recent years, specific primers for the amplification of Apicomplexa in reptiles have been developed, and have been shown to be very useful, considerably increasing the sensitivity of detection (e.g., Ujvari et al. 2004, Harris et al. 2011, Maia et al. 2011, Tomé et al. 2012). In this study we demonstrate that these primers can also amplify other protists, namely Proteromonas (Stramenopiles, see Cavalier-Smith and Chao 2006). 
At present, limited molecular data is available for the genus Proteromonas; there are currently only data from P. lacertae for the mitochondrion genome (Pérez-Brocal et al. 2010) and from rRNA genes (Leipe et al. 1996, Hoevers and Snowden 2005). Phylogenetic studies have shown that Proteromonas and other members of the Slopalinida are related with the genus Blastocystis, a group of protozoan parasites of medical and veterinary importance (e.g., Tan 2004, Kostka et al. 2007).

\section{Materials and methods}

DNA was extracted from blood or tail tissue using standard High Salt methods (Sambrook et al. 1989), DNeasy Blood \& Tissue kit (Qiagen), or Speedtools Tissue DNA extraction kit (Biotools). Primers used were HepF300 and HepR900 (Ujvari et al. 2004) targeting part of the $18 \mathrm{~S}$ rRNA gene and PCR cycling consisted of $94^{\circ} \mathrm{C}-30 \mathrm{sec}, 60^{\circ} \mathrm{C}-30 \mathrm{sec}, 72^{\circ} \mathrm{C}-1 \mathrm{~min}$ (35 cycles) (see Harris et al. 2011 for more details). Negative and positive controls were run with each reaction. PCR products were electrophoresed on $2 \%$ agarose gels, stained using sybr safe or gel red and visualized using a UV-transilluminator. The positive PCR products were purified and sent for direct sequencing (Macrogen Inc). All amplifications were sequenced in both directions. Sequence chromatograms were checked manually and assembled using Geneious v. 5.6.4 (Biomatters Ltd.). We then performed a similarity analysis using the Basic Local Alignment Search Tool (BLAST) to find the best match (E-value $\leq 10-8$ ) for the sequences against published sequences in GenBank (http://www.ncbi.nlm.nih.gov). All sequences obtained matched the single $P$. lacertae $16 \mathrm{~S}$ - like rRNA gene sequence available (U37108) with 98\% similarity. The new sequences have been submitted to GenBank (accession numbers JX276957-JX276959).

To assess phylogenetic relationships, these sequences were aligned with the following sequences retrieved from GenBank: 1) representatives of Proteromonadidae family: $P$. lacertae (U37108), Karotomorpha sp. (DQ431242 and DQ431243); 2) representatives of the Opalinidae family: Protoopalina japonica (AB175929), Protoopalina intestinalis (AY576545), Opalina ranarum (AF146089); 3) Blastocystis sp. (AY135412) and Blastocystis pythoni (AY266472), which were used as outgroups for rooting the phylogenetic trees following Kostka et al. (2007). Alignments were performed using the MUSCLE algorithm (Drummond et al. 2012) using default parameters implemented in Geneious 5.6.4. The final alignment consisted of 11 sequences of 575 bp. Two different phylogenetic analyses (Maximum Likelihood and Bayesian Inference) were conducted. Maximum Likelihood (ML) analysis included random sequence addition (100 replicate heuristic searches), and support for nodes was estimated using the bootstrap technique (Felsenstein 1985) with 1000 replicates, using PhyML 3.0 (Guindon et al. 2010). The AIC criteria carried out in jModeltest 0.1.1 (Posada 2008) were used to choose the model of evolution employed $(\mathrm{GTR}+\mathrm{I}+\mathrm{G})$. Bayesian analysis was implemented using Mr. Bayes 3.1 (Huelsenbeck and Ronquist 2001) with parameters estimated as part of the analysis. The analysis was run for $5 \times 10^{6}$ generations, saving one tree each 1000 generations. The log-likelihood values of the sample point were plotted against the generation time and all the trees prior to reaching stationary were discarded. Remaining trees were combined in a 50\% majority consensus tree (Fig. 1). Upon collection, slides were air-dried and later fixed with methanol and stained with Giemsa following Telford

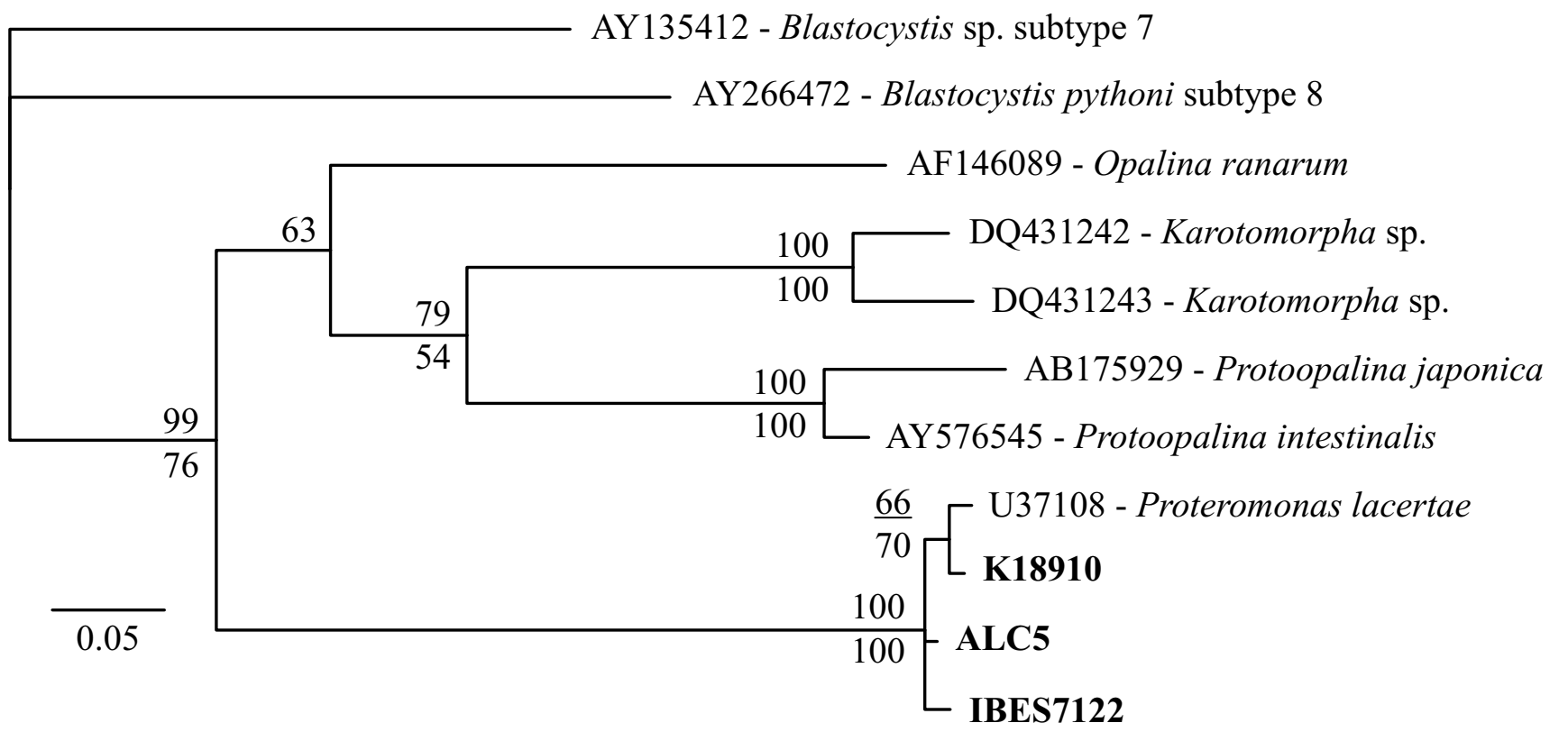

Fig. 1. Bayesian Inference tree of the new Proteromonas sequences obtained in this study together with sequences retrieved from GenBank. Support for the Bayesian and for ML analysis are given above and below the nodes, respectively 
Table I. Comparison between P. lacertae sequence retrieved from GenBank (U37108) together with the Apicomplexa specific primers used in this study. Mismatches are indicated in bold

\begin{tabular}{lll}
\hline & HEP300F & HEP900R \\
\hline Primer & GTTTCTGACCTATCAGCTTTCGACG & GTCAGAGGTGAAATTCTTAGATTTG \\
U37108 & GTTTCTGCCCTATCAGCTTTCGATG & GTCAGAGGTGAAATTCAAGGATTTA \\
\hline
\end{tabular}

(2009). Microscopy was conducted at 400x and 1000x magnification using an Olympus CX41 microscope with an in-built digital camera (SC30).

\section{Results}

During routine molecular screening of Apicomplexa parasites in reptiles, we have amplified and sequenced a portion of the Proteromonas $18 \mathrm{~S}$ rRNA gene in three reptile samples out of around 600 reptile samples analysed, less than $1 \%$ prevalence. These samples belong to three different lizard genera: two from the family Lacertidae (tail tissue from Acanthodactylus erythrurus from Spain, sample ALC5, and blood drop from Darevskia armeniaca from Armenia, sample K18910), and one from the family Gekkonidae (blood drop from Pristurus carteri from Oman, sample IBES7122). Of these samples, a blood smear was only available for IBES7122, in which flagellate stages of Proteromonas could be identified (Fig. 2). A comparison between U37108 and the Apicomplexa specific primers used in this study (HEP300F and HEP900R) showed only two and four mismatches, respectively (Table I). Our phylogenetic analysis using sequences obtained in this study together with previously published sequences of other related Stramenopiles, confirms these Proteromonas 18S rRNA gene sequences to be closely related to Proteromonas lacertae (see Fig. 1). In the conserved regions of the alignment, the three sequences obtained in this study differ from the $P$. lacertae retrieved from GenBank (Leipe et al. 1996) by one (K18910), two (ALC5) and four positions (IBES7122). Additionally, there is a short (20 nucleotides) hyper-variable region in which all samples differ considerably in sequence and length, and where is variability within the single host sample for K18910 and IBES7122. The sequence from GenBank also has an ambiguity (A or $G$ ) in the same region.

\section{Discussion}

In this study, we show that sequences of unexpected parasites can be amplified from host samples (blood and tissue) using general procedures to detect hemoparasites. We have amplified and sequenced a segment of the Proteromonas 18S rRNA gene from three lizard host genera using specific primers for the amplification of Apicomplexa. The taxonomic position of these sequences was further confirmed by our phylogenetic analyses. Although our phylogeny is limited due to scarcity of molecular data on these protists, the relationships are congruent with those obtained in previous published works (Kostka et al. 2004, 2007; Nishi et al. 2005; Hoevers and Snowden 2005). The position of Karotomorpha and of opalinids, agrees
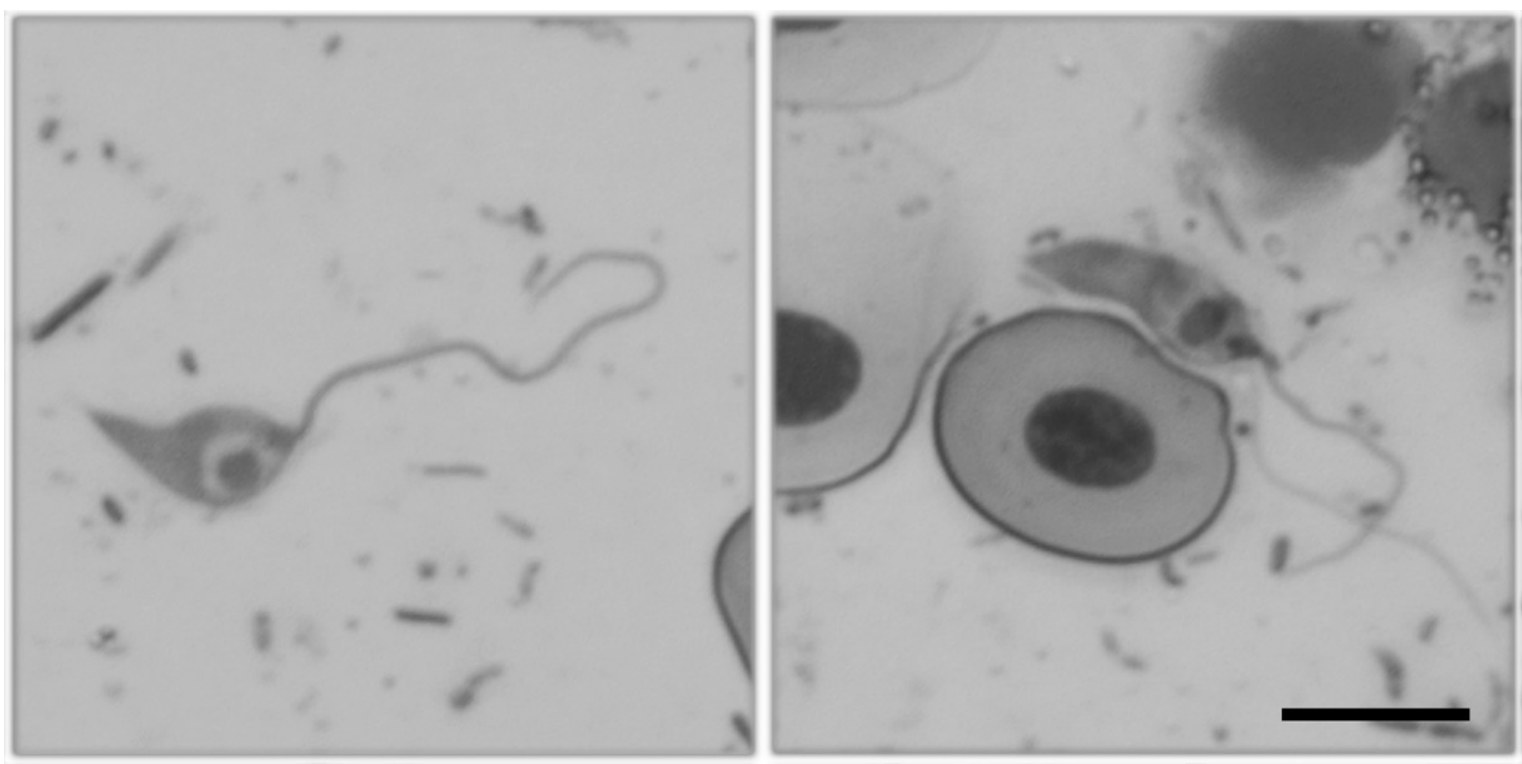

Fig. 2. Microscopy picture of the blood smear of sample IBES7122 from P. carteri showing flagellate stages of Proteromonas. Scale bar $=0.01 \mathrm{~mm}$ 
indeed with the topology obtained by Kostka et al. (2007) that showed the family Proteromonadidae as paraphyletic, with the genus Karotomorpha being more closely related to opalinids than to the genus Proteromonas.

Interestingly, Proteromonas is a genus of obligate anaerobe protists that live as commensals in the colon of lizards. However, in this study we detected Proteromonas-like organisms in blood and tail-tissue samples. One hypothesis is that faecal runoff (containing cysts) could be present on the skin of the reptile tissue sampled. Indeed, lizards often defecate when being processed, so there is the possibility that the parasites present in the faeces may be transferred to the blood drops or remain in the skin of the tail tip. Alternatively, cysts or flagellate stages of these organisms may be naturally present in the blood stream (the latter shown in Fig. 2), or occasionally the trophic stages may be able to invade tissues. Other unexpected parasites have also recently been reported from blood samples, including Eimeria, which is also typically detected in faecal samples (Harris et al. 2012). This clearly warrants further investigation.

Regardless of the source, our study demonstrates the perils of molecular diagnosis protocols in parasitological studies and the need of cross-validation procedures between and within methodologies. First, the primers used were originally designed in a study where 100 pythons were found to have $100 \%$ prevalence of presumably Hepatozoon spp. based solely on PCR (Ujvari et al. 2004). However, these primers are actually less specific and can amplify other distantly related protists (e.g., Proteromonas) or even fungi (Tomé et al. 2012), as well as various apicomplexans such as Eimeria and Sarcocystis (Harris et al. 2012), which could pose an important bias for infection estimates. Nonetheless, the fact that primers are less specific may also open up new possibilities in providing relevant information on other parasitic organisms, as long as these are confirmed through sequencing and phylogenetic analyses. It therefore should be stressed that it is important not to rely solely on PCR amplifications when screening for infections; PCR products should be sequenced to confirm identification of the parasite detected. This will not only avoid false positives, if other unrelated parasites are amplified, but also has the potential to provide valuable information on the diversity and evolutionary relationships of poorly-known or less common parasites.

Acknowledgements. JPMCM is supported by a Fundação para a Ciência e a Tecnologia (FCT) PhD grant (SFRH/BD/74305/2010) and co-financed by FSE and POPH and EU. EG-D was supported by a Juan de la Cierva contract from the Ministerio de Educación y Ciencia, Spain. Financial support was provided by project ERGPARIS-276838 from the European Commission. Thanks to our colleagues from CIBIO who helped with the fieldwork and to the people and entities that made it possible to obtain samples from the different countries. Thanks also to the two anonymous reviewers for their helpful comments on an earlier draft of this manuscript.

\section{References}

Amo L., López P., Martín J. 2005. Prevalence and intensity of haemogregarine blood parasites and their mite vectors in the common wall lizard, Podarcis muralis. Parasitology Research, 96, 378-381. DOI: 10.1007/s00436-005-1354-2.

Austin C.C., Perkins S.L. 2006. Parasites in a biodiversity hotspot: a survey of hematozoa and a molecular phylogenetic analysis of Plasmodium in New Guinea pigs skinks. Journal of Parasitology, 92, 770-777. DOI: 10.1645/GE-693R.1.

Cavalier-Smith T., Chao E.E.-Y. 2006. Phylogeny and megasystematics of phagotrophic heterokonts (kingdom Chromista). Journal of Molecular Evolution, 62, 388-420. DOI: 10.1007/ s00239-004-0353-8.

Dollahon N.R., Janovy J., Jr. 1971. Insect flagellates from feces and gut contents of four genera of lizards. Journal of Parasitology, 57, 1130-1132.

Drummond A.J., Ashton B., Buxton S., Cheung M., Cooper A., Duran C., Field M., Heled J., Kearse M., Markowitz S., Moir R., Stones-Havas S., Sturrock S., Thierer T., Wilson A. 2012. Geneious v5.6. Available from http://www.geneious.com.

Felsenstein J. 1985. Confidence intervals on phylogenies: an approach using the bootstrap. Evolution, 39, 783-791.

Guindon S., Dufayard J.F., Lefort V., Anisimova M., Hordijk W., Gascuel O. 2010. New algorithms and methods to estimate maximum-likelihood phylogenies: assessing the performance of PhyML 3.0. Systematic Biology, 59, 307-321, DOI: 10.1016/j.ympev.2011.08.029.

Harris D.J., Maia J.P.M.C., Perera A. 2011. Molecular characterization of Hepatozoon species in reptiles from the Seychelles. Journal of Parasitology, 97, 106-110. DOI: 10.1645/GE2470.1 .

Harris D.J., Maia J.P.M.C., Perera A. 2012. Molecular survey of Apicomplexa in Podarcis wall lizards detects Hepatozoon, Sarcocystis and Eimeria species. Journal of Parasitology, 98, 592-597. DOI: 10.1645/JP-GE-2908R2.

Hoevers J.D., Snowden K.F. 2005. Analysis of the ITS region and partial ssu and lsu rRNA genes of Blastocystis and Proteromonas lacertae. Parasitology, 131, 187-196. DOI:10.1017/ S0031182005007596.

Huelsenbeck J.P., Ronquist F. 2001. MRBAYES: Bayesian inference of phylogenetic trees. Bioinformatics, 17, 754-755.

Janakidevi K. 1961a. A new species of Chilomastix alexeieff, 1912 (Protozoa: Retortamonadines, Grassé, 1952) from the Indian lizard. Zeitschrift für Parasitenkunde, 20, 563-567.

Janakidevi K. 1961b. A new species of Hexamastix (Protozoa) parasitic in the spiny-tailed lizard, Uromastyx hardwickii. Zeitschrift für Parasitenkunde, 21, 151-154.

Kostka M., Hampl V., Cepicka I., Flegr J. 2004. Phylogenetic position of Protoopalina intestinalis based on SSU rRNA gene sequence. Molecular Phylogenetics and Evolution, 33, 220-224. DOI: 10.1016/j.ympev.2004.05.009.

Kostka M., Cepicka I., Hampl V., Flegr J. 2007. Phylogenetic position of Karotomorpha and paraphyly of Proteromonadidae. Molecular Phylogenetics and Evolution, 43, 1167-1170. DOI: 10.1016/j.ympev.2006.11.002.

Krishnamurthy R. 1968. A new flagellate of the genus Proteromonas Kunstler, 1883, from an Indian lizard. Parasitology, 58, 231534. DOI: 10.1017/S0031182000028833.

Leipe D.D., Tong S.M., Goggin C.L., Slemenda S.B., Pieniazek N.J., Sogin M.L. 1996. 16S-like rDNA sequences from Developayella elegans, Labyrinthuloides haliotidis, and Proteromonas lacertae confirm that the stramenopiles are a primarily heterotrophic group. European Journal of Protistology, 32, 449-458. DOI: 10.1016/S0932-4739(96)800 04-6. 
Maia J.P.M.C., Harris D.J., Perera A. 2011. Molecular survey of Hepatozoon species in lizards from North Africa. Journal of $\mathrm{Pa}$ rasitology, 97, 513-517. DOI: 10.1645/GE-2666.1.

Merino S., Vásquez R.A., Martínez J., Celis-Diez J.L., Gutiérrez-Jiménez L., Ippi S., Sánchez-Monsalvez I., La Puente J.M. 2009. Molecular characterization of an ancient Hepatozoon species parasitizing the 'living fossil' marsupial 'Monito del Monte' Dromiciops gliroides from Chile. Biological Journal of the Linnean Society, 98, 568-576. DOI: 10.1111/j.10958312.2009.01302.x.

Moody A. 2002. Rapid Diagnostic Tests for Malaria Parasites. Clinical Microbiology Reviews, 15, 66-78. DOI: 10.1128/CMR. 15.1.66-78.2002.

Nishi A., Ishida K., Endoh H. 2005. Reevaluation of the evolutionary position of opalinids based on $18 \mathrm{~S}$ rDNA, and $\alpha$ - and $\beta$ tubulin gene phylogenies. Journal of Molecular Evolution, 60, 695-705. DOI: 10.1007/s00239-004-0149-x.

Pérez-Brocal V., Shahar-Golan R., Clark C.G. 2010. A linear molecule with two large inverted repeats: the mitochondrial genome of the stramenopile Proteromonas lacertae. Genome Biology and Evolution, 2, 257-266. DOI: 10.1093/gbe/evq 015.

Posada D. 2008. jModelTest: Phylogenetic model averaging. Molecular Biology and Evolution, 25, 1253-1256. DOI: 10.1093/ molbev/msn083.

Roca V., Galdón M.A. 2010. Haemogregarine blood parasites in the lizards Podarcis bocagei (Seoane) and P. carbonelli (PérezMellado) (Sauria: Lacertidae) from NW Portugal. Systematic Parasitology, 75, 75-79. DOI: 10.1007/s11230-009-9206-6.

Rubini A.S., dos S. Paduan K., Cavalvante G.G., Ribolla P.E.M., O’Dwyer L.H. 2005. Molecular identification and characterization of canine Hepatozoon species from Brazil. Parasitology Research, 97, 91-93. DOI: 10.1007/s00436-005-1383-x.

Sambrook J., Fritsch E.F., Maniatis T. 1989. Molecular cloning: a laboratory manual. Cold Spring Harbor Press, Cold Spring Harbor, New York, 545 pp.
Saratchandra B., Narasimhamurti C.C. 1980a. A new species of Proteromonas, P. grassei n. sp. from the gut of Hemidactylus prashadi Smith. Proceedings: Animal Sciences, 89, 293-295. DOI: $10.1007 / \mathrm{BF} 03179171$.

Saratchandra B., Narasimhamurti C.C. 1980b. Proteromonas waltairensis n. sp. from the wall lizard, Hemidactylus prashadi Smith. Indian Journal of Parasitology, 4, 73-75.

Tan K.S.W. 2004. Blastocystis in humans and animals: new insights using modern methodologies. Veterinary Parasitology, 126, 121-144. DOI: 10.1016/j.vetpar.2004.09.017.

Telford S.R., Jr. 1970. A comparative study of endoparasitism among some southern California lizard populations. American Midland Naturalist, 83, 516-554.

Telford S.R., Jr., Bursey C.R. 2003. Comparative parasitology of squamate reptiles endemic to scrub and sand hills communities of north-central Florida, U.S.A. Comparative Parasitology, 70, 172-181. DOI: 10.1654/4060.

Telford S.R. 2009. Hemoparasites of the Reptilia: Color atlas and text. CRC Press, Boca Raton, Florida, 394 pp.

Tomé B., Maia J.P.M.C., Harris D.J. 2012. Hepatozoon infection prevalence in four snake genera: influence of diet, prey parasitaemia levels or parasite type? Journal of Parasitology, 98, xxx-xxx, DOI: 10.1645/GE-3111.1.

Ujvari B., Madsen T., Olsson M. 2004. High prevalence of Hepatozoon spp. (Apicomplexa, Hepatozoidae) infection in water pythons (Liasisfuscus) from tropical Australia. Journal of Parasitology, 90, 670-672. DOI: 10.1645/GE-204R.

Vardo A.M., Wargo A.R., Schall J.J. 2005. PCR detection of lizard malaria parasites: prevalence of Plasmodium infections with low-level parasitemia differs by site and season. Journal of Parasitology, 91, 1509-1511. DOI: 10.1645/GE-589R.1.

Wenrich D.H. 1947. Culture experiments on intestinal flagellates. III. Species from Amphibians and Reptiles. Journal of Parasitology, 33, 62-70.

Wood W.F. 1935.Some observations on the intestinal protozoa of Californian lizards. Journal of Parasitology, 21, 165-174. 